\title{
Dissecting Genuine and Deceptive Kudos: The Case of Online Hotel Reviews
}

\author{
Snehasish Banerjee, Alton Y. K. Chua \\ Wee Kim Wee School of Communication \& Information \\ Nanyang Technological University \\ 31 Nanyang Link, SCI Building, Singapore 637718
}

\begin{abstract}
As users continue to rely on online hotel reviews for making purchase decisions, the trend of posting deceptive reviews to heap praises and kudos is gradually becoming a wellestablished e-business malpractice. Conceivably, it is not trivial for users to distinguish between genuine and deceptive kudos in reviews. Hence, this paper identifies three linguistic cues that could offer telltale signs to distinguish between genuine and deceptive reviews. These linguistic cues include readability, genre and writing style. Drawing data from a publicly available secondary dataset, results indicate that readability and writing style of reviews offer useful clues to distinguish between genuine and deceptive reviews. Specifically, genuine reviews could be more readable and less hyperbolic compared with deceptive entries. With respect to review genre however, the differences were largely blurred. The implications of the findings for theory and practice are highlighted.
\end{abstract}

Keywords-e-business; user-generated content; online reviews; opinion spam; readability; genre; writing style

\section{INTRODUCTION}

Users increasingly rely on online reviews for making purchase decisions. In particular, they are often inclined to trust positive reviews, which are meant to applaud products and services, as confirming evidence before making a choice [1]. Furthermore, positive reviews are usually more abundant in review websites than those with either negative or mixed opinions [2]. As a result, it is conceivable that praises and kudos in reviews could significantly impact users' purchase decisions.

However, users need to exercise caution while interpreting positive reviews. Since positive reviews have the potential to boost sales of a given product or service, they offer adequate incentives for organizations to indulge in e-business malpractices such as opinion spamming [3,4]. For the purpose of this paper, opinion spamming involves posting deceptive reviews containing fictitious praises and kudos with a deliberate attempt to resemble genuine entries. Such a practice is gradually growing into one of the popular e-business tactics among businesses $[5,6,7]$. Hence, consumers could be misled while making purchase decisions.

While it may not be easy to distinguish between genuine and deceptive positive reviews, there could be subtle differences in ways they are written. Hence, this paper seeks to uncover linguistic nuances unique to genuine and deceptive kudos in reviews.
To achieve the objective, it examines authenticity of positive reviews based on three linguistic cues, namely, readability, genre and writing style. Readability refers to the effort and expertise required on the part of users to comprehend the meaning of reviews [7, 8, 9]. Genre refers to the degree to which reviews are informative, which in turn, could influence their distribution of part-of-speech (POS) tags $[10,11,12]$. Writing style refers to authoring approaches such as the use of affective cues, perceptual words and future tense $[13,14,15,16]$.

This paper is significant for both theory and practice. On the theoretical front, it builds on the research areas related to genuine and deceptive reviews through a linguistic analysis. It confirms that nuances in readability and writing style of positive reviews help distinguish between genuine and deceptive entries with reasonable accuracy. In contrast, it suggests that differences between the two could be largely blurred in terms of genre. On the practical front, this paper calls for caution in interpreting reviews, and honesty while posting entries.

The remainder of this paper is structured as follows. The next section presents the related literature. This is followed by the details of the dataset, as well as the operationalization and analysis procedures. The results are presented next. Thereafter, three key findings gleaned from the results are discussed. Finally, the paper concludes with notes on limitations, implications, and directions for future research.

\section{LITERATURE REVIEW}

The profusion of Web 2.0 has made user-generated content ubiquitous. A specific form of user-generated content that has exponentially grown in popularity and acceptance includes online reviews, which are meant to evaluate products and services. Specifically, reviews for hotels are widely used by users prior to making a booking [17, 18]. They are often perceived as being more genuine and credible vis-à-vis thirdparty advertisements [19]. Hence, it is no wonder that more than some $80 \%$ consumers tend to choose their holiday accommodation based on properties that had been widely applauded in reviews [18].

Users' growing proclivity for hotel reviews thus provides an ideal opportunity for businesses to indulge in opinion spamming. Specifically, positive deceptive reviews heaping praises and kudos are considered priceless. After all, such entries could result in significant financial gains and fames for 
businesses [5, 6, 20]. Hence, it is not surprising that posting positive deceptive reviews is fast becoming a well-established e-business malpractice [7].

To aggravate the problem, deceptive reviews are deliberately written to appear genuine. As a result, the lines between them could often be blurred. Nonetheless, drawing from prior studies, this paper argues that even though genuine and deceptive reviews are not easily distinguishable, there could be subtle telltale signs in terms of their readability, genre, and writing style $[7,16,21]$. These three linguistic cues are explained in greater details as follows.

\section{A. Readability}

Readability refers to the effort and expertise required on the part of users to comprehend the meaning of reviews [7, 8, 9]. Since genuine and deceptive reviews are written in different contexts, the readability of the two could be different from each other.

Writing genuine reviews is cognitively less challenging than articulating deceptive entries [22]. Moreover, individuals performing a writing task with a high cognitive load tend to write more lucid language than those performing the same task with a lower cognitive load [23]. Hence, deceptive reviews could be more lucid compared with genuine reviews. Stated otherwise, genuine reviews could be less readable vis-à-vis deceptive ones.

However, another school of thought suggests that genuine reviews could be more readable compared with deceptive entries. This is because when users browse reviews, they not only read the entries but also gauge the intelligence of their contributors [24, 25]. Too simplistic reviews might suggest incompetence of the respective contributors in writing sophisticated reviews. Hence, deceptive reviews could be deliberately written using sophisticated language to showcase contributors' competence. This in turn might take a toll on readability.

\section{B. Genre}

Genre refers to the degree to which reviews are informative $[10,11,12]$. Writing genuine reviews requires articulating real experiences. On the other hand, writing deceptive reviews requires articulating imaginary experiences that did not occur in reality. Texts written based on real experiences could differ in terms of their genre from accounts based on imagined experiences [26].

There are four genres of text, namely, conversational, taskoriented, informative and imaginative [12]. Among these, genuine reviews could be more informative while deceptive reviews could lean towards being imaginative [11]. Texts of informative genre differ from those of imaginative genre in their distribution of POS tags [10, 11, 12]. Specifically, informative texts contain more adjectives, articles, nouns, and prepositions. In contrast, imaginative texts contain more adverbs, verbs, and pronouns [10, 24].

Among pronouns, personal pronouns in the form of selfreferences has attracted special attention among the scholarly community. On the one hand, spammers could feel the pangs of conscience while writing deceptive reviews [27]. As a result, they might use fewer personal pronouns to dissociate themselves from their deceptive comments. On the other hand, spammers could also be enthused by the prospect of deceiving others easily. With great resolve to conceal their deception, it is also possible for them to deliberately enrich deceptive reviews with personal pronouns [16, 28].

\section{Writing Style}

Writing style refers to authoring approaches used in reviews. For the purpose of this paper, writing style entails the use of affective cues, perceptual words, and future tense [13, $14,15,16]$. Deceptive reviews could be replete with positive affective cues as a form of exaggeration to create a lasting impact among readers in the online community [29].

Besides, users' physical experiences with hotels are affected by their sensory perceptions [30]. For instance, users' opinion about a hotel could be a function of visual cues such as artwork and aural cues such as music [15]. These cues are reflected in reviews through the use of perceptual words. Conceivably, genuine reviews written after real post-trip experience could be rich in perceptual words.

Additionally, given that positive reviews could favorably impact future sales and revenues of a given hotel [13, 20], deceptive reviews might be articulated not only to describe past experiences in the hotel, but also to express future desires of staying in the same hotel again. Such a writing style might suggest that the positive experiences described in the deceptive reviews are far from being ephemeral. On the other hand, genuine reviews could simply describe past experiences. Hence, they might contain fewer future tense compared with deceptive reviews.

\section{METHODS}

\section{A. Dataset}

A major challenge that hinders research on genuine and deceptive reviews is the difficulty in ascertaining ground truth [31]. After all, it is challenging to validate what is genuine, and what is deceptive in the first place [32]. This has often led scholars to alternatively employ heuristic annotation approaches. For example, [3] deemed duplicate or near duplicate reviews as deceptive ignoring that duplications might at times stem from technical glitches or human errors. Moreover, [33] labeled reviews as either genuine or deceptive with the help of some annotators, who had read a few articles on ways to identify spam. Despite being intuitive, the validity of such heuristic annotation approaches is questionable.

This paper therefore draws ground truth from a publicly available secondary dataset of 800 positive reviews [11]. Specifically, the dataset comprises 400 genuine reviews, and 400 deceptive reviews uniformly distributed across 20 popular hotels in Chicago. Thus, for every hotel, the dataset contained 20 genuine reviews $(20$ hotels $\mathrm{x} 20$ genuine reviews $=400$ genuine reviews altogether), and 20 deceptive reviews (20 hotels $\mathrm{x} 20$ deceptive reviews $=400$ deceptive reviews altogether).

This dataset was selected for analysis due to two reasons. First, it is one of the recent works on linguistic differences between genuine and deceptive reviews. Of late, it has been 
widely cited by the scholarly community [e.g., 21, 34, 35]. Second, to the best of our knowledge, it is the only publicly available dataset of genuine and deceptive positive reviews till date.

\section{B. Operationalization and Analysis}

Readability was operationalized based on four metrics, namely, Gunning-Fog Index (FOG) [36], Coleman-Liau Index (CLI) [37], Automated-Readability Index (ARI) [38], and Flesch-Kincaid Grade Level (FKG) [39, 40]. Each metric employs a set of unique constants and depend on factors such as number of characters per word, number of words per sentence, and number of syllables per word. More detailed description about these metrics can be found in works such as [8], [9] and [41]. A Java program was written to compute these metrics. Lower values for the readability metrics suggest greater readability. Among the four metrics, FOG and CLI specifically indicate complexity, while ARI and FKG are proxies for reading difficulty [8]. Therefore, FOG and CLI scores for every review were averaged to create a composite index for complexity. Likewise, ARI and FKG scores for every review were averaged to create a composite index for reading difficulty. Finally, review readability was measured in terms of two indicators, namely, (1) complexity, and (2) reading difficulty.

Review genre was operationalized on the basis of the POS tag distributions in reviews. Specifically, the following eight POS tags were considered: (1) adjective, (2) article, (3) noun, (4) preposition, (5) adverb, (6) verb, (7) pronoun, and (8) personal pronoun. While the first four are expected to be higher in genuine reviews, the next four could be higher in deceptive reviews $[10,12,16]$. The fractions of each of these POS tags in reviews were computed using Stanford Parser's POS tagger [42].

Review writing style was operationalized as the proportion of (1) positive cues, (2) perceptual words, and (3) future tense used in reviews. These indicators were measured using the Linguistic Inquiry and Word Count (LIWC) software [43]. It is an automated text analysis tool that offers reliable dictionaries to compute such linguistic indicators.
To sum up, this paper includes a total of 13 independent variables (IVs) for analysis as follows: the two readability indicators, the eight POS tags, and the three writing style indicators. The categorical dependent variable (DV) comprises review authenticity. Since this paper seeks to examine review authenticity as a function of readability, genre and writing style, the DV was dummy-coded such that 1 indicates genuine reviews and 0 denotes deceptive reviews. Given its dichotomous nature, binomial logistic regression was used for data analysis [44]. The coefficients of logistic regression estimate the odds ratio, indicating the extent to which the IVs in the model could predict review authenticity.

To diagnose potential problems of multicollinearity in the logistic regression model, the variance inflation factors (VIF) for all the 13 IVs were examined. The VIF values were found to be less than 10, suggesting that multicollinearity did not exist [45]. Another potential problem of logistic regression is the presence of outliers in the solution [46]. In particular, cases with standardized residual values of above 2.5 or below -2.5 could be problematic [47]. Only one out of 800 reviews was found to have exceeded the acceptable threshold, and was retained for the analysis.

After analysis, the performance of the logistic regression model was probed using Omnibus test, and Hosmer-Lemeshow goodness-of-fit test. The extent to which the model could account for the variability in the dependent variable was examined using two pseudo- $\mathrm{R}^{2}$ measures, namely, Cox and Snell $\mathrm{R}^{2}$, as well as Nagelkerke $\mathrm{R}^{2}$ [47]. Finally, the ability of the model to differentiate between genuine and deceptive reviews was checked using 10 -fold cross-validation. This facilitates checking the model's stability in distinguishing between genuine and deceptive reviews for unknown datasets.

\section{RESULTS}

Table I presents the non-parametric inter-correlations among the variables involved in the analysis. Variables 1 through 13 represent the 13 IVs, while variable 14 comprises the DV, namely, review authenticity. With respect to the DV, 10 of the 13 IVs had statistically significant correlations.

TABLE.I. SPEARMAN NON-PARAMETRIC INTER-CORRELATIONS BETWEEN VARIABLES INVOLVED IN THE ANALYSIS

\begin{tabular}{|c|c|c|c|c|c|c|c|c|c|c|c|c|c|c|}
\hline Variables & 1 & 2 & 3 & 4 & 5 & 6 & 7 & 8 & 9 & 10 & 11 & 12 & 13 & 14 \\
\hline 1 Complexity & 1 & & & & & & & & & & & & & \\
\hline 2 Reading diff. & $.92^{*}$ & 1 & & & & & & & & & & & & \\
\hline 3 Adjective & $.09^{*}$ & .02 & 1 & & & & & & & & & & & \\
\hline 4 Article & -.01 & .01 & -.02 & 1 & & & & & & & & & & \\
\hline 5 Noun & $.14^{*}$ & .07 & .02 & $.07^{*}$ & 1 & & & & & & & & & \\
\hline 6 Preposition & $.15^{*}$ & $.20^{*}$ & $-.27^{*}$ & -.05 & .06 & 1 & & & & & & & & \\
\hline 7 Adverb & $-.20^{*}$ & $-.19^{*}$ & .06 & $-.19^{*}$ & $-.33^{*}$ & $-.26^{*}$ & 1 & & & & & & & \\
\hline 8 Verb & $-.34^{*}$ & $-.31^{*}$ & $-.10^{*}$ & $-.11^{*}$ & $-.50^{*}$ & $-.31^{*}$ & $.26^{*}$ & 1 & & & & & & \\
\hline 9 Pronoun & $-.16^{*}$ & $-.08^{*}$ & $-.40^{*}$ & $-.31^{*}$ & $-.48^{*}$ & -.04 & $.12^{*}$ & $.39^{*}$ & 1 & & & & & \\
\hline 10 Pers. pronoun & $-.13^{*}$ & $-.07^{*}$ & $-.42^{*}$ & $-.28^{*}$ & $-.41^{*}$ & .01 & $.11^{*}$ & $.33^{*}$ & $.86^{*}$ & 1 & & & & \\
\hline 11 Positive cues & $.09^{*}$ & -.01 & $.39^{*}$ & $-.03^{*}$ & -.02 & $-.29^{*}$ & $.14^{*}$ & -.02 & $-.13^{*}$ & $-.14^{*}$ & 1 & & & \\
\hline 12 Percep. words & .05 & .02 & $.10^{*}$ & $-.09^{*}$ & -.01 & -.01 & -.03 & -.02 & .02 & .02 & $.11^{*}$ & 1 & & \\
\hline 13 Future tense & -.01 & -.01 & -.05 & $-.12^{*}$ & $-.18^{*}$ & $-.12^{*}$ & $.24^{*}$ & $.17^{*}$ & $.14^{*}$ & $.12^{*}$ & .04 & .01 & 1 & \\
\hline $14 \mathrm{DV}$ & $-.19^{*}$ & $-.18^{*}$ & $.12^{*}$ & .06 & $.15^{*}$ & .02 & -.03 & $-.10^{*}$ & $-.31^{*}$ & $-.33^{*}$ & $-.11^{*}$ & $-.11^{*}$ & $-.14^{*}$ & 1 \\
\hline
\end{tabular}


Descriptive statistics of the dataset are presented in Table II and Table III. In particular, Table II provides mean, standard deviation, minimum and maximum of the 13 IVs for the full dataset of 800 reviews (full), the subset comprising 400 genuine reviews (genu), and the subset containing 400 deceptive reviews (decep). Thereafter, range as well as first, second and third quartiles of the IVs are presented in Table III.

TABLE.II. MEAN, Standard DeViation, Minimum and MaXimum of The 13 Ivs

\begin{tabular}{|l|l|l|l|l|l|l|l|l|l|l|l|l|l|}
\hline \multirow{2}{*}{ IVs } & Mean & \multicolumn{9}{l|}{ Standard Deviation } & \multicolumn{2}{l|}{ Minimum } & \multicolumn{2}{l|}{ Maximum } \\
\cline { 2 - 16 } & full & genu & decep & full & genu & decep & full & genu & decep & full & genu & decep \\
\hline Complexity & 9.26 & 8.94 & 9.59 & 2.42 & 2.67 & 2.09 & 3 & 3 & 4 & 27.5 & 27.5 & 18.5 \\
\hline Reading diff. & 6.78 & 6.53 & 7.04 & 3.62 & 4.39 & 2.63 & .5 & .5 & 1.5 & 45.5 & 45.5 & 23.5 \\
\hline Adjective & 10.48 & 10.88 & 10.09 & 3.33 & 3.47 & 3.14 & 2 & 2 & 3 & 27 & 27 & 23 \\
\hline Article & 10.10 & 10.26 & 9.95 & 2.75 & 2.90 & 2.57 & 0 & 0 & 2 & 18 & 18 & 18 \\
\hline Noun & 26.90 & 27.67 & 26.13 & 4.97 & 5.18 & 4.64 & 15 & 15 & 15 & 44 & 44 & 43 \\
\hline Preposition & 12.13 & 12.19 & 12.07 & 3.02 & 2.93 & 3.12 & 2 & 3 & 2 & 20 & 20 & 20 \\
\hline Adverb & 5.11 & 5.00 & 5.23 & 2.60 & 2.36 & 2.82 & 0 & 0 & 0 & 18 & 15 & 18 \\
\hline Verb & 12.24 & 11.87 & 12.60 & 3.24 & 3.17 & 3.27 & 2 & 2 & 3 & 24 & 20 & 24 \\
\hline Pronoun & 10.59 & 9.38 & 11.80 & 3.96 & 3.63 & 3.91 & 0 & 0 & 1 & 23 & 21 & 23 \\
\hline Pers. pronoun & 7.04 & 5.96 & 8.12 & 3.31 & 2.92 & 3.33 & 0 & 0 & 0 & 17 & 14 & 17 \\
\hline Positive cues & 6.89 & 6.63 & 7.15 & 2.95 & 2.99 & 2.89 & 0 & 0 & 1 & 19 & 19 & 19 \\
\hline Percep. words & 2.08 & 1.90 & 2.26 & 1.59 & 1.52 & 1.65 & 0 & 0 & 0 & 9 & 9 & 8 \\
\hline Future tense & 0.84 & 0.71 & 0.98 & 0.87 & 0.77 & 0.95 & 0 & 0 & 0 & 5 & 4 & 5 \\
\hline
\end{tabular}

TABLE.III. RANGE AND QUARTILES OF THE 13 IVS

\begin{tabular}{|l|l|l|l|l|l|l|l|l|l|l|l|l|l|}
\hline \multirow{2}{*}{ IVs } & \multicolumn{4}{l}{ Range } & \multicolumn{3}{l|}{ First Quartile } & \multicolumn{2}{l|}{ Second Quartile } & \multicolumn{2}{l|}{ Third Quartile } \\
\cline { 2 - 15 } & full & genu & decep & full & genu & decep & full & genu & decep & full & genu & decep \\
\hline Complexity & 24.5 & 24.5 & 14.5 & 8 & 7.5 & 8 & 9 & 8.5 & 9.5 & 10.5 & 10 & 11 \\
\hline Reading diff. & 45 & 45 & 22 & 5 & 4.5 & 5.5 & 6.5 & 6 & 6.5 & 8 & 7.5 & 8.5 \\
\hline Adjective & 25 & 25 & 20 & 8 & 9 & 8 & 10 & 10 & 10 & 12 & 13 & 12 \\
\hline Article & 18 & 18 & 16 & 8 & 8.3 & 8 & 10 & 10 & 10 & 12 & 12 & 12 \\
\hline Noun & 29 & 29 & 28 & 24 & 24 & 23 & 26 & 27 & 26 & 30 & 31 & 29 \\
\hline Preposition & 18 & 17 & 18 & 10 & 10 & 10 & 12 & 12 & 12 & 14 & 14 & 14 \\
\hline Adverb & 18 & 15 & 18 & 3 & 3 & 3 & 5 & 5 & 5 & 7 & 6 & 7 \\
\hline Verb & 22 & 18 & 21 & 10 & 10 & 10 & 12 & 12 & 12 & 14 & 14 & 15 \\
\hline Pronoun & 23 & 21 & 22 & 7 & 7 & 9 & 11 & 9 & 12 & 13 & 12 & 14 \\
\hline Pers. pronoun & 17 & 14 & 17 & 4 & 4 & 6 & 7 & 6 & 8 & 9 & 8 & 11 \\
\hline Positive cues & 19 & 19 & 18 & 5 & 5 & 5 & 6 & 6 & 7 & 8 & 8 & 8.75 \\
\hline Percep. words & 9 & 9 & 8 & 1 & 1 & 1 & 2 & 2 & 2 & 3 & 3 & 3 \\
\hline Future tense & 5 & 4 & 5 & 0 & 0 & 0 & 1 & 1 & 1 & 1 & 1 & 1 \\
\hline
\end{tabular}


For the logistic regression model, result of the Omnibus test indicates acceptable performance of the model $\left(\chi^{2}=206.74\right.$; $\mathrm{df}$ $=13 ;-2 \log$ likelihood $=902.29 ; \mathrm{p}<0.001)$. The HosmerLemeshow goodness-of-fit test indicated a non-significant result $\left(\chi^{2}=7.11 ; \mathrm{df}=8 ; \mathrm{p}=0.53\right)$, which suggests that the model fits well with the data. Cox and Snell $\mathrm{R}^{2}$ was 0.27 while Nagelkerke $\mathrm{R}^{2}$ was 0.36 . Thus, around $27 \%$ to $36 \%$ of the variability in review authenticity could be explained by the model. Using 10-fold cross-validation, the model accurately predicted 281 of the 400 genuine reviews, and hence had a genuine review prediction accuracy of $70.25 \%$. On the other hand, it could accurately predict 283 of the 400 deceptive reviews, and hence had a deceptive review prediction accuracy of $70.75 \%$. Overall, it recorded an accuracy of $70.50 \%$.

Results further indicate that the two readability indicators, namely, complexity and difficulty could significantly predict if reviews were genuine or deceptive. In particular, complexity was negatively related to review authenticity $[\beta=-0.75, \operatorname{Exp}(\beta)$ $=0.47, \mathrm{p}<0.001]$. The higher the value of complexity for a given review, the lower was its likelihood to be genuine. Put differently, genuine reviews had lower values for complexity compared with deceptive reviews, suggesting that the former is linguistically less complex than the latter. However, reading difficulty was positively related to review authenticity $[\beta=$ $0.37, \operatorname{Exp}(\beta)=1.45, \mathrm{p}<0.001]$. In other words, genuine were generally more difficult to be read compared with deceptive entries.

The discordant finding between complexity and reading difficulty could be vestige of the uniqueness of the four readability metrics. Furthermore, it supports the argument that the two readability indicators, namely, complexity and reading difficulty, do not necessarily imply each other [8]. However, to the best of our knowledge, not much research hitherto has disinterred the nuances between complexity and reading difficulty in the context of genuine and deceptive reviews. To further tease out nuances, the factors that affect FOG, CLI, ARI and FKG were delved deeper.

These four readability metrics are primarily affected by three constituent factors, namely, (1) average characters per word, (2) average words per sentence, and (3) average syllables per word $[8,9,41]$. To disinter variations between genuine and deceptive reviews based on the three factors, independent samples t-tests were performed. In terms of average characters per word, genuine reviews $(\mathrm{M}=4.40, \mathrm{SD}=0.28)$ did not significantly differ from deceptive reviews $(\mathrm{M}=4.40, \mathrm{SD}=$ 0.30 ). With respect to average words per sentence too, there was no significant difference between genuine $(\mathrm{M}=14.46, \mathrm{SD}$ $=9.72)$ and deceptive reviews $(\mathrm{M}=15.07, \mathrm{SD}=5.26)$. However in terms of average syllables per word, there was a significant difference between genuine reviews $(\mathrm{M}=1.40, \mathrm{SD}$ $=0.10)$ and deceptive reviews $(\mathrm{M}=1.44, \mathrm{SD}=0.12)$ $[\mathrm{t}(779.85)=4.53, \mathrm{p}<0.001]$. Given that genuine reviews used significantly lower number of syllables per word compared with deceptive reviews, the former seems to fare better in terms of readability.

Among the eight POS tags, articles, pronouns and personal pronouns turned out to be significant predictors of review authenticity. All three were negatively related to the DV as follows: articles $[\beta=-0.12, \operatorname{Exp}(\beta)=0.89, \mathrm{p}<0.01]$, pronouns $[\beta=-0.11, \operatorname{Exp}(\beta)=0.89, p<0.05]$, and personal pronouns $[\beta$ $=-0.19, \operatorname{Exp}(\beta)=0.82, p<0.001]$. In other words, reviews with fewer articles, pronouns and personal pronouns were more likely to be genuine. On the other hand, reviews that comprised more articles, pronouns and personal pronouns were more likely to be deceptive.

With respect to writing style, all the three metrics, namely, the use of positive cues, perceptual words and future tense, emerged as significant predictors of review authenticity. All three were negatively related to the DV as follows: positive cues $[\beta=-0.10, \operatorname{Exp}(\beta)=0.90, p<0.01]$, perceptual words $[\beta$ $=-0.15, \operatorname{Exp}(\beta)=0.86, p<0.01]$, and future tense $[\beta=-0.30$, $\operatorname{Exp}(\beta)=0.74, p<0.01]$. Thus, it appears that deceptive reviews were generally more richly embellished with positive cues, perceptual words and future tense compared with genuine reviews. Table IV summarizes the extent to which the $13 \mathrm{IVs}$ in the model could predict review authenticity.

TABLE.IV. RESULTS OF THE LOGISTIC REGRESSION MODEL

\begin{tabular}{|c|c|c|c|c|c|}
\hline $\begin{array}{l}\text { Linguistic } \\
\text { Cues }\end{array}$ & IVs & $\boldsymbol{\beta}$ & SE & Wald & $\operatorname{Exp}(\beta)$ \\
\hline \multirow{2}{*}{ Readability } & Complexity $^{* * *}$ & -0.75 & 0.10 & 52.16 & 0.47 \\
\hline & Reading difficulty ${ }^{* * *}$ & 0.37 & 0.07 & 31.87 & 1.45 \\
\hline \multirow[t]{8}{*}{ Genre } & Adjective & 0.02 & 0.04 & 0.50 & 1.02 \\
\hline & Article $^{* *}$ & -0.12 & 0.04 & 10.69 & 0.89 \\
\hline & Noun & -0.02 & 0.02 & 0.39 & 0.98 \\
\hline & Preposition & -0.04 & 0.03 & 1.25 & 0.96 \\
\hline & Adverb & -0.04 & 0.04 & 1.31 & 0.95 \\
\hline & Verb & -0.06 & 0.03 & 2.67 & 0.95 \\
\hline & Pronoun ${ }^{*}$ & -0.11 & 0.04 & 6.50 & 0.89 \\
\hline & Personal pronoun $^{* * * *}$ & -0.19 & 0.05 & 15.01 & 0.82 \\
\hline \multirow[t]{3}{*}{ Writing Style } & Positive cues ${ }^{* * *}$ & -0.10 & 0.03 & 9.52 & 0.90 \\
\hline & Perceptual words ${ }^{* *}$ & -0.15 & 0.05 & 7.54 & 0.86 \\
\hline & Future tense ${ }^{* *}$ & -0.30 & 0.10 & 8.52 & 0.74 \\
\hline Pseudo- $\mathrm{R}^{2}$ & \multicolumn{5}{|c|}{0.27 (Cox and Snell), 0.36 (Nagelkerke) } \\
\hline Accuracy & \multicolumn{5}{|c|}{$70.50 \%$ (10-fold cross-validation) } \\
\hline
\end{tabular}

$$
{ }^{* * *} \mathrm{P}<0.001,{ }^{* *} \mathrm{P}<0.01,{ }^{*} \mathrm{P}<0.05
$$

\section{DISCUSSION}

Three key findings could be gleaned from the results. First, in terms of readability, genuine reviews were more readable than deceptive reviews. For example, a readable genuine review in the dataset indicated, "...the hotel is centrally located ... is less than a block away. perfect location! the suites are huge with comfy beds... also they have a free dinner..." In contrast, a less readable deceptive review stated, "...boasts a modern fitness center that feature free weights, a cardio room, dry saunas, as well as, masseurs...elegant with a touch of historic decor...breath-taking view of Chicago, as well as, had an in-room mini-bar, terry-cloth bath robes, over-sized desks, high-speed internet access, and a 37-inch Hi Def LCD Television..." This finding is consistent with prior research which suggested that deceptive content could be less readable than the genuine counterpart [21, 48, 49]. Unlike users articulating genuine experiences, spammers were overly ostentatious in reflecting their competence in writing sophisticated reviews $[24,25]$. That could be why deceptive reviews comprised significantly higher syllables per word as compared with genuine entries. Interpreting this finding on the basis of self-presentation effect [50], users writing genuine 
reviews appear less motivated than spammers to make ostentation of linguistic competence.

Second, in terms of genre, genuine and deceptive reviews appeared to share similar levels of informativeness. For example, an informative genuine review pointed, "...large room with 2 double beds and 2 bathrooms, The TV was Ok, a 27' CRT Flat Screen... The breakfast is charged, 20 dollars...close to metro station..." Likewise, an informative deceptive review expressed, "...located in the heart of...has a 24 hour business center providing high-speed internet access, fax, and photocopying services...in-room mini-bar... and a 37inch Hi Def LCD Television..." Prior research suggests that genuine and deceptive reviews could be informative and imaginative respectively $[11,12]$. While the former could contain more adjectives, articles, nouns and prepositions, the latter could be richer in adverbs, verbs, pronouns and personal pronouns. However, only articles, pronouns and personal pronouns could significantly distinguish between genuine and deceptive reviews. Furthermore, the finding that reviews with fewer articles were more likely to be genuine contradicts literature on text genre $[10,12]$. The dominance of personal pronouns in deceptive reviews over genuine entries reflects the lack of guilt among spammers. Although prior research expects them to feel guilty and use less self-references to dissociate themselves from deceptive content [26, 27, 51], such a phenomenon was generally inconspicuous. A deceptive review rich in personal pronouns stated, "...I came with very little...my deluxe room supplied me with everything that I needed...I will be back..." This suggests that spammers could be adept enough to blur the lines between genuine and deceptive reviews with respect to text genre.

Third, in terms of writing style, genuine reviews appeared less hyperbolic compared with deceptive ones. Consistent with extant literature [16, 29], deceptive reviews seemed to include significantly more positive affective cues than genuine reviews. For example, a deceptive review pointed, "The hotel was very nice; Service was great, everyone was very friendly. The room was very elegant... had a great experience...pleasant staff...I left here well rested and happy." In order to steer users' impression on hotels towards a positive light, deceptive reviews seemed to contain more perceptual words than genuine entries. This was perhaps deliberately done to appeal to the sensory perceptions of the online community $[15,30]$. A highly perceptual deceptive review illustrated, "...view from my windows was stunning, as I looked out I could see the beautiful...room had a nice airy feel but was also warm...bed was very comfortable..." The excessive use of future tense in deceptive reviews might have been used to assure that the positive experiences at the hotel were not ephemeral. After all, positive reviews could be highly influential in stimulating future sales and revenues of a given hotel $[13,20]$. A deceptive review rich in future tense expressed, "... will leave you absolutely relaxing... will leave you wanting to visit the moment you leave."

\section{CONCLUSION}

As users continue to rely on online hotel reviews for making purchase decisions, the trend of posting deceptive reviews to heap praises and kudos is gradually becoming a well-established e-business malpractice. Hence, this paper attempted to distinguish between genuine and deceptive reviews using linguistic analysis. In particular, it investigated the extent to which linguistic differences between genuine and deceptive reviews in terms of readability, genre, and writing style could predict review authenticity. Drawing data from a publicly available secondary dataset, results indicate that readability and writing style of reviews are useful clues to distinguish between genuine and deceptive reviews. Specifically, genuine reviews could be more readable and less hyperbolic compared with deceptive entries. With respect to review genre however, the differences were largely blurred.

It should be acknowledged that the findings of the paper are somewhat constrained by the dataset used for analysis. For one, it comprised only positive reviews. Even though it facilitated distinguishing between genuine and deceptive kudos in reviews, the findings are not generalizable to negative reviews that are meant to criticize hotels, or mixed reviews that highlight both merits and demerits of hotels. Moreover, the reviews were meant for some popular hotels in Chicago. Hence, it is unknown if the findings could be extrapolated to all types of hotels located in various geographical locations. Moreover, the dataset size of 800 reviews was not overly large. This could have resulted in inaccurate findings. Nonetheless, this paper does offer implications for both theory and practice.

On the theoretical front, this paper augments prior studies such as $[3,7,11]$ by conducting a linguistic analysis of genuine and deceptive reviews. It demonstrates that readability and writing style of reviews could significantly distinguish between genuine and deceptive reviews. While genuine reviews could be more readable vis-à-vis deceptive entries, the former could be articulated with a less hyperbolic writing style. In terms of genre however, this paper demonstrates that genuine and deceptive reviews are equally informative. This finding is at odds with prior studies such as [52] and [26], which expected genuine reviews to be more informative than the deceptive counterpart.

On the practical front, this paper serves as an eye-opener pointing that all positive reviews should not be trusted. Prior research suggests that when users browse reviews, they could be tempted to trust positive entries that are generally abundant $[1,2,53]$. However, not every positive review is necessarily authentic [7, 11]. Hence, users need to exercise caution. They could lean on the findings of this paper to conjecture which reviews are likely to be genuine accounts of post-trip experiences and hence, can be relied for travel planning. Based on the findings, moderators of review websites could automatically recommend reviews that are potentially genuine and flag off those that are likely to be deceptive. The findings can thus play a significant role in preventing users from being victims of deceptive opinion spamming. This will aid more informed travel planning, thereby mitigating hotels' e-business malpractice of promoting themselves through deceptive kudos in reviews.

This paper further offers a few potential directions for future research. For one, the dataset could be expanded beyond reviews for hotels to include those for other products and services such as consumer electronics, or downloadable 
applications. Another possible direction could include analysis of the extent to which linguistic differences predict review authenticity across positive, negative and mixed reviews. Such studies could help extend the theoretical boundaries of this paper. Moreover, the finding that genuine and deceptive reviews are equally informative is significant for further research. Spammers are increasingly becoming smarter to blur the lines between the two. As they learn the patterns to mimic genuine reviews, it is important for the scholarly community to catch up. Perhaps in due course of time, linguistic analysis alone might no longer be sufficient to distinguish between genuine and deceptive reviews. In this vein, this paper serves to pique further scholarly inquiry into this research theme from aspects beyond linguistic analysis.

\section{REFERENCES}

[1] Y. Pan, and J. Q. Zhang, "Born unequal: A study of the helpfulness of user-generated product reviews," Journal of Retailing, vol. 87, 2011, pp. 598-612.

[2] N. Hu, J. Zhang, and P. A. Pavlou, "Overcoming the J-shaped distribution of product reviews," Communications of the ACM, vol. 52, 2009, pp. 144-147.

[3] N. Jindal, and B. Liu, "Opinion spam and analysis," Proceedings of the International Conference on Web Search and Web Data Mining, 2008, pp. 219-230.

[4] D. Fallis, D. "A conceptual analysis of disinformation," Proceedings of the iConference, 2009, Chapel Hill, NC. Available at http://ideals.illinois.edu/bitstream/handle/2142/15205/fallis_disinfo1.pdf

[5] E. Allen, "Dear staff. We could do with some positive comments: Hotel boss is caught telling his workers to post fake reviews on TripAdvisor," MailOnline (2012, October 9). Internet: http://www.dailymail.co.uk/new s/article-2214974/Hotel-boss-caught-telling-workers-post-fake-reviewsTripAdvisor.html [Dec. 26, 2013].

[6] C. Fernandez, "Tripadvisor bribes: Hotel owners offer free rooms in return for glowing reviews," MailOnline (2011, July 11). Internet: http://www.dailymail.co.uk/travel/article-2013391/Tripadvisor-Hotelowners-bribe-guests-return-good-reviews.html [Jun. 5, 2013].

[7] N. Hu, I. Bose, N. S. Koh, and L. Liu, "Manipulation of online reviews: An analysis of ratings, readability, and sentiments," Decision Support Systems, vol. 52, 2012, pp. 674-684.

[8] N. Korfiatis, E. García-Bariocanal, and S. Sánchez-Alonso, "Evaluating content quality and helpfulness of online product reviews: The interplay of review helpfulness vs. review content," Electronic Commerce Research and Applications, vol. 11, 2012, pp. 205-217.

[9] B. L. Zakaluk, and S. J. Samuels, Readability: Its past, present and future. Newark: International Reading Association, 1988.

[10] G. Leech, P. Rayson, and A. Wilson, Word frequencies in written and spoken English: Based on the British National Corpus. London: Longman, 2001.

[11] M. Ott, Y. Choi, C. Cardie, and J. T. Hancock, "Finding deceptive opinion spam by any stretch of the imagination," Proceedings of the Association for Computational Linguistics, 2011, pp. 309-319.

[12] P. Rayson, A. Wilson, and G. Leech, "Grammatical word class variation within the British National Corpus sampler," Language and Computers, vol. 36, 2001, pp. 295-306.

[13] W. Duan, B. Gu, and A. B. Whinston, "Do online reviews matter? An empirical investigation of panel data," Decision Support Systems, vol. 45, 2008, pp. 1007-1016.

[14] H. C. Lench, S. A. Flores, and S. W. Bench, "Discrete emotions predict changes in cognition, judgment, experience, behavior, and physiology: A meta-analysis of experimental emotion elicitations," Psychological Bulletin, vol. 137, 2011, pp. 834-855.

[15] I. Y. Lin, "Evaluating a servicescape: The effect of cognition and emotion," International Journal of Hospitality Management, vol. 23, 2004, pp. 163-178.
[16] K. H. Yoo, and U. Gretzel, "Comparison of deceptive and truthful travel reviews," ICT in Tourism, 2009, pp. 37-47.

[17] J. Fotis, D. Buhalis, and N. Rossides, "Social media impact on holiday travel planning: The case of the Russian and the FSU markets," International Journal of Online Marketing, vol. 1, 2011, pp. 1-19.

[18] K. L. Sidali, H. Schulze, and A. Spiller, "The impact of online reviews on the choice of holiday accommodations," ICT in Tourism, 2009, pp. 87-98.

[19] S. Senecal, and J. Nantel, "The influence of online product recommendations on consumers' online choices," Journal of Retailing, vol. 80, 2004, pp. 159-169.

[20] L. S. Chen, and J. Y. Lin, "A study on review manipulation classification using decision tree," International Conference on Service Systems and Service Management, 2013, pp. 680-685.

[21] T. Ong, M. Mannino, and D. Gregg, "Linguistic characteristics of shill reviews," Electronic Commerce Research and Applications, vol. 13, 2014, pp. 69-78.

[22] M. Zuckerman, B. M. DePaulo, and R. Rosenthal, "Verbal and nonverbal communication of deception," Advances in Experimental Social Psychology, vol. 14, 1981, pp. 1-59.

[23] J. K. Burgoon, and T. Qin, "The dynamic nature of deceptive verbal communication," Journal of Language and Social Psychology, vol. 25, 2006, pp. 76-96.

[24] L. Connors, S. M. Mudambi, and D. Schuff, "Is it the review or the reviewer? A multi-method approach to determine the antecedents of online review helpfulness," Proceedings of the IEEE Hawaii International Conference on System Sciences, 2011, pp. 1-10.

[25] P. F. Wu, H. van der Heijden, and N. Korfiatis, "The influences of negativity and review quality on the helpfulness of online reviews," Proceedings of the International Conference on Information Systems, 2011, pp. 1-10.

[26] A. Vrij, K. Edward, K. P. Roberts, and R. Bull, "Detecting deceit via analysis of verbal and nonverbal behavior," Journal of Nonverbal Behavior, vol. 24, 2000, pp. 239-264.

[27] A. Vartapetiance, L. Gillam, "II don't know where he is not': Does deception research yet offer a basis for deception detectives?," Proceedings of Computational Approaches to Deception Detection, 2012, pp. 5-14.

[28] S. Godin, All Marketers Are Liars. New York: Portfolio, 2005.

[29] C. Maurer, and S. Schaich, "Online customer reviews used as complaint management tool," ICT in Tourism, 2011, pp. 499-511.

[30] H. R. Schiffman, Sensation and Perception, 5th Edition. New York: Wiley, 2001.

[31] S. Gokhman, J. Hancock, P. Prabhu, M. Ott, and C. Cardie, "In search of a gold standard in studies of deception," Proceedings of the Workshop on Computational Approaches to Deception Detection, 2012, pp. 23-30.

[32] G. Wu, D. Greene, and P. Cunningham, "Merging multiple criteria to identify suspicious reviews," Proceedings of the ACM Conference on Recommender systems, 2010, pp. 241-244.

[33] F. Li, M. Huang, Y. Yang, and X. Zhu, "Learning to identify review spam," Proceedings of the International Joint Conference on Artificial Intelligence, 2011, pp. 2488-2493.

[34] J. Parapar, D. E. Losada, and A. Barreiro, "Combining psycholinguistic, content-based and chat-based features to detect predation in chatrooms," Journal of Universal Computer Science, vol. 20, 2014, pp. 213-239.

[35] H. Sun, A. Morales, and X. Yan, "Synthetic review spamming and defense," Proceedings of the International Conference on Knowledge Discovery and Data Mining, 2013, pp. 1088-1096.

[36] R. Gunning, The technique of clear writing. New York: McGraw-Hill, 1952.

[37] M. Coleman, and T. L. Liau, "A computer readability formula designed for machine scoring," Journal of Applied Psychology, vol. 60, 1975, pp. 283-284.

[38] E. A. Smith, and R. J. Senter, Automated readability index, AMRL-TR66-22. Wright-Patterson AFB, Ohio: Aerospace Medical Division, 1967.

[39] R. F. Flesch, How to test readability. New York: Harper, 1951. 
[40] J. P. Kincaid, R. P. Fishburne Jr, R. L. Rogers, and B. S. Chissom, Derivation of new readability formulas (automated readability index, fog count and flesch reading ease formula) for navy enlisted personnel, No. RBR-8-75, Naval Technical Training Command, Millington TN Research Branch, 1975.

[41] W. H. DuBay, The Principles of Readability, 2004. Internet: http://files.eric.ed.gov/fulltext/ED490073.pdf [Sep. 30, 2014]

[42] D. Klein, and C. D. Manning, "Accurate unlexicalized parsing," Proceedings of the Association for Computational Linguistics, 2003, pp. 423-430.

[43] J. W. Pennebaker, C. K. Chung, M. Ireland, A. Gonzales, and R. J. Booth, "The development and psychometric properties of LIWC 2007," Austin, TX: LIWC.net, 2007.

[44] J. F. Hair, R. E. Anderson, R. L. Tatham, and W. C. Black, Multivariate data analysis, 5th edition. New Jersey: Prentice Hall, 1998.

[45] R. Myers, Classical and Modern Regression with Applications. Boston: Duxbury Press, 1990.

[46] B. G. Tabachnick, and L. S. Fidell, Using multivariate statistics, 3rd Edition. New York: Harper Collins, 1996.

[47] J. Pallant, SPSS survival manual: A step by step guide to data analysus using SPSS for Windows (version 12). Sydney: Allen \& Unwin, 2005.
[48] R. L. Daft, and R. H. Lengel, "Information richness: A new approach to managerial behavior and organizational design," in Research in organizational behavior, L. L. Cummings, and B.M. Staw, Eds. Homewood, Illinois: JAI Press, 1984, pp. 191-233.

[49] K. Moffitt, and M. B. Burns, "What does that mean? Investigating obfuscation and readability cues as indicators of deception in fraudulent financial reports," Proceedings of Americas Conference on Information Systems, 2009, pp. 399:1-10.

[50] M. R. Leary, Self-presentation: Impression management and interpersonal behavior. Wisconsin: Brown \& Benchmark, 1995.

[51] A. Vrij, K. Edward, and R. Bull, "Stereotypical verbal and nonverbal responses while deceiving others," Personality and Social Psychology Bulletin, vol. 27, 2001, pp. 899-909.

[52] M. L. Newman, J. W. Pennebaker, D. S. Berry, and J. M. Richards, "Lying words: Predicting deception from linguistic styles," Personality and Social Psychology Bulletin, vol. 29, 2003, pp. 665-675.

[53] P. A. Pavlou, and A. Dimoka, "The nature and role of feedback text comments in online marketplaces: Implications for trust building, price premiums, and seller differentiation," Information Systems Research, vol. 17, 2006, pp. 392-414. 